\title{
COMBINED BENDING WITH TENSION OF ISOTROPIC PLATE WITH CRACK CONSIDERING CRACK BANKS CONTACT AND PLASTIC ZONES AT ITS TOPS
}

\author{
Heorgij SULYM*, Viktor OPANASOVYCH ${ }^{* *}$, Mykola SLOBODIAN** Oksana BILASH ${ }^{* * *}$
}

\author{
*Department of Mechanics and Applied Computer Science Application, Faculty of Mechanical Engineering, \\ Bialystok University of Technology, ul. Wiejska 45 C, 15-351 Bialystok, Poland \\ ${ }^{*}$ Faculty of Mechanics and Mathematics, Department of Mechanics, The Ivan Franko National University of Lviv, \\ Universytetska Street, 1, Lviv, 79000, Ukraine \\ ${ }^{* * *}$ Faculty Training Specialists Battle (Operational) Software, Department of Engineering Mechanics (Weapons and Equipment of Military \\ Engineering Forces), Hetman Petro Sahaidachnyi National Army Academy, Heroes of Maidan Street, 32, Lviv, Ukraine
}

sulym@pb.edu.pl, viktor.opanasovych@Inu.edu.ua, slobkolia@gmail.com, oksana.opanasovych@gmail.com

received 13 March 2017, revised 11 May 2018, accepted 15 May 2018

\begin{abstract}
Stress-strain state of isotropic plate with rectilinear through-crack at combined action of bending and tension, realized by applying distributed forces and bending moments at infinity, the vectors of which are parallel and perpendicular to the crack, is investigated. Under the influence of the internal stress the crack faces contacts on area of constant width near the upper base of plate, and plastic zones forms in its tips. Using methods of the theory of complex variables, complex potentials plane problem of elasticity theory and the classical theory of plates bending, solving of the problem is reduced to the set of linear conjugation problems and their analytical solution is built in a class of functions of limited plastic zones in the crack tips. The conditions of existence of the solution of the problem in these terms are determined. Using Treska plasticity conditions in the form of surface layer or the plastic hinge, the length of plastic zone and crack opening displacement are found analytically. Their numerical analysis for various parameters of the problem is conducted.
\end{abstract}

Key words: Isotropic Plate, Bending, Tension, Crack, Treska Plasticity Conditions

\section{INTRODUCTION}

The problems of bending and tension of plates with cracks are very common. In the article of Chen et al. (2005) the problems of periodic system of cracks in an infinite plate are investigated. For solving of the problems singular integral equation is used. In the article of Shi (2015) a high-accurate solving procedure that describes the effect of interacting of double-periodic rectangular systems of cracks is proposed. The bending of a thin infinite plate with a linear crack and a rigid inclusion of random shape is analyzed in the work of Wang et al. (2000). The solution is obtained in integral form by using the Green function of a singular dislocation. In the work of Boukellif et al. (2014) the method for cracks detection in plates is presented. This method allows determining parameters of crack, such as length, location and angles relatively to a reference coordinate system as so as calculation of stress intensity factors (SIF).

In the article of Prawoto (2012) classical fracture mechanics approach is used for calculation of the plastic zones that appears near cracks in heterogeneous or composite materials. In research of Unger D.J. (2007) instead of Tresca plasticity theory the Dugdale model of plasticity is used for a static crack. In the work of García-Collado et al. (2017) a numerical analysis of influence of plasticity, caused by crack closure, that uses the method of crack propagation in combination with cyclic plasticity in the crack tip. The Reissner's problems of bend of plates with cracks are given in the works of Guimaraes et al. (2009), Samaan et al. (2015), Sulym et al. (2011).

Research of stress-strain state at biaxial bending or combined bending and tension of isotropic plate with through-crack considering crack faces contact both on the crack line and in the region of constant width near one of the basis of the plate, without considering the plastic zones in the crack tip are represented in the works of Shackyj et al. (1989, 1995, 2004a, 2004b), Slepyan et al. (1995), Papargyri-Pegiou et al. (1995), Opanasovych (2007, 2008), excluding papers of Alfavicka (2015) and Opanasovych et al. (2015), where such consideration is done. In this article is studied the case of combined bending and tension of isotropic plate with through crack, faces of which are in contact on the area of constant width under the action of distributed forces and bending moments, applied at infinity, the vectors of which, are parallel and perpendicular to the crack faces, and considering plastic zones in the crack tip, where Treska plasticity conditions in the form of surface layer or the plastic hinge are satisfied. Using the method of complex potentials the solving of the problem is reduced to linear conjugation problems and the explicit expressions for complex potentials of plane problem of elasticity theory and the classical theory of bending of plates in the class of functions of limited plastic zones at the crack tips are found. The length of the plastic zone and crack opening displacement is found analytically and its numerical analysis with various parameters is done. In the particular case the known results are obtained.

\section{FORMULATION OF THE PROBLEM}

Let us consider an infinite isotropic plate of thickness $2 h$, weakened by rectilinear through-crack of length $2 l$. Crack faces 
and the front surface are free from external loads. At infinity the plate is subjected to bending moments $M_{x}^{\infty}, M_{y}^{\infty}$ and tension forces $p$ and $q$, applied symmetrically to the crack (see Fig. 1). The crack faces under the action of external loads are in smooth contact on area of constant width $h_{1}$ near the upper base of the plate (Opanasovych et al., 2008) and in the tips of crack strip-like plastic zones are forming, where Treska plasticity conditions are satisfied in the form of surface layer or the plastic hinge (Kushnir et al., 2003, Kyrjan et al. 2007).

We now introduce a Cartesian coordinate system $0 x y \tilde{z}$ with the origin in the center of crack, so the middle surface of the plate coincides with $O x y$ coordinate plane and $O x$ axis coincides with the crack line. We denote the crack length with plastic zones of length $b$ as $2 d$, the crack contour as $L$, and plastic zones as $L_{1}$, so $\tilde{L}=L+L_{1}$.

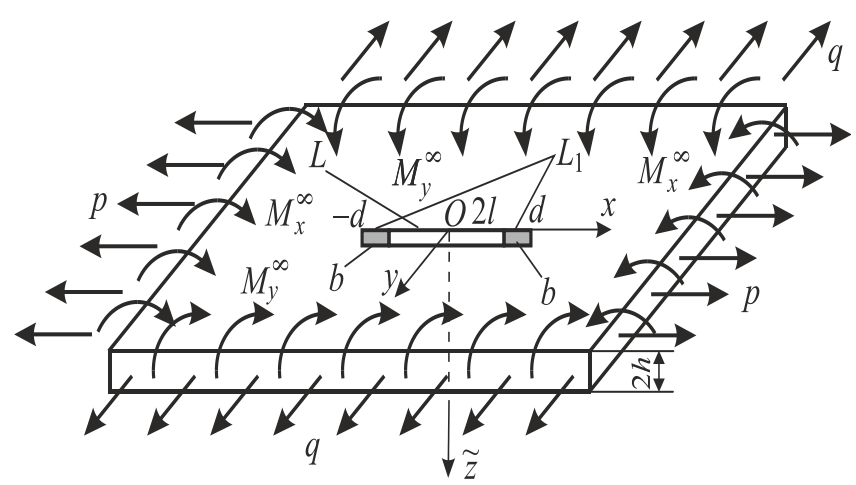

Fig. 1. Loading scheme of a plate and location of a crack with plastic zones

Since the crack faces are in contact, the solution of the problem can be divided into two: the plane problem of elasticity theory with unknown normal stresses, applied to crack faces, and the problem of bend with unknown bending moments applied to crack faces. In case of plane problem the constant normal stress $\sigma_{0}$ is acting in the plastic zones and in case of the problem of bend bending moment $\mathrm{M}_{0}$ is acting. To describe bend we use the technical theory of bend of plates. For the crack faces in the plastic zones we have following boundary conditions:

$\sigma_{\overline{y y}}^{ \pm}=\sigma_{y y}, \sigma_{\overline{x y}}^{ \pm}=0, x \in L$,

$M_{y}^{ \pm}=M_{\mathrm{y}}, \mathrm{P}_{y}^{ \pm} y=0, x \in L$,

$M_{\mathrm{y}}=\beta N, \partial_{x}[v]+\alpha\left[\partial_{x y}^{2} w\right]=0, x \in L$,

$\sigma_{y y}^{ \pm}=\sigma_{0}, \sigma_{x y}^{ \pm}=0, x \in L_{1}$,

$M_{\bar{y}}^{ \pm}=M_{0}, P_{y}^{ \pm}=0, x \in L_{1}$,

where: $\mathrm{N}=-2 \mathrm{~h} \sigma_{\mathrm{yy}}, \mathrm{N}-$ crack faces contact force $(\mathrm{N}>0)$; $\sigma_{\mathrm{yy}}, \sigma_{\mathrm{xy}}-$ stress tensor components; $\mathrm{u}, \mathrm{v}-$ displacement vector components at the plane problem (Musheviashvili, 1966); $\mathrm{P}_{\mathrm{y}}$ - generalized Kirchhoff cross-force; $\mathrm{M}_{\mathrm{y}}$ - bending moment; $\mathrm{w}-$ deflection of the plate (Prusov, 1975); in the second formula (3) we introduce the notation $[\rho(x)]=\rho^{+}(x)-\rho^{-}(x), "+"$ and "-" signs correspond to limit value of an appropriate value at $\mathrm{y} \rightarrow \pm 0 ; \quad[\mathrm{f}]=\mathrm{f}^{+}-\mathrm{f}^{-} ; \quad \alpha$ and $\beta-$ constant, which are determined by the formula (Opanasovych, 2007):

$\beta=1-\gamma / 3, \alpha=0.5\left[1+(1-\gamma)^{2}\right], \gamma=h_{1} / h$.

\section{CONSTRUCTION OF THE SOLUTION OF THE PROBLEM}

Let us introduce the complex potentials $\Phi(z)$ and $\Omega(z)$ (Musheviashvili, 1966) for plane problem and $\Phi_{*}(z)$ and $\Omega_{*}(z)$ (Prusov, 1975) - for bend, and then use the dependencies

$$
\begin{aligned}
& \sigma_{y y}-i \sigma_{x y}=\Phi(z)+\Omega(\bar{z})+(z-\bar{z}) \overline{\Phi^{\prime}(z)} \\
& 2 \mu \partial_{x}(u+i v)=\kappa \Phi(z)-\Omega(\bar{z})-(z-\bar{z}) \overline{\Phi^{\prime}(z)} \\
& \partial_{x} g=\Phi_{*}(z)-\Omega_{*}(\bar{z})+(z-\bar{z}) \overline{\Phi_{*}^{\prime}(z)} \\
& f=\tilde{\kappa} \Phi_{*}(z)+\Omega_{*}(\bar{z})-(z-\bar{z}) \overline{\Phi_{*}^{\prime}(z)}
\end{aligned}
$$

where: $\mu=\mathrm{E}[2(1+v)]^{-1}$ - shear module, $\mathrm{E}-$ Young's module, $\mathrm{v}$ - Poisson's ratio of the material of the plate, $\mathrm{i}=$ $\sqrt{-1}, \kappa=(3-v) /(1+v), \tilde{\kappa}=(3+v) /(1-v), z=x+$ iy, $\quad g=\partial_{\mathrm{x}} \mathrm{w}+\mathrm{i} \partial_{\mathrm{y}} \mathrm{w}, \quad \mathrm{f}=\left(\mathrm{M}_{\mathrm{y}}+\mathrm{ic} \mathrm{c}^{\prime}+\mathrm{i} \int_{0}^{\mathrm{x}} \mathrm{P}(\varepsilon) \mathrm{d} \varepsilon\right) / \mathrm{m}$, $\mathrm{m}=-\mathrm{D}(1-\mathrm{v}), \mathrm{c}^{\prime}-$ unknown real constant, $\mathrm{D}=\frac{2 \mathrm{Eh}^{3}}{3\left(1-\mathrm{v}^{2}\right)}-$ cylindrical rigidity of plate.

Complex potential for large $|z|$ can be represented as (Musheviashvili, 1966, Prusov, 1975):

$$
\begin{aligned}
& \Phi(z)=\Gamma+O\left(1 / z^{2}\right), \Omega(z)=\Gamma+\Gamma^{\prime}+O\left(1 / z^{2}\right), \\
& \Phi_{*}(z)=\tilde{\Gamma}+O\left(1 / z^{2}\right), \Omega_{*}(z)=-\tilde{\Gamma}-\widetilde{\Gamma}^{\prime}+O\left(1 / z^{2}\right),
\end{aligned}
$$

where: $\Gamma=\frac{1}{4}(q+p), \Gamma^{\prime}=\frac{1}{2}(q-p), \tilde{\Gamma}=-\frac{M_{x}^{\infty}+M_{y}^{\infty}}{4 D(1+v)}$, $\widetilde{\Gamma}^{\prime}=-\frac{M_{\mathrm{y}}^{\infty}-M_{\mathrm{x}}^{\infty}}{2 m}$.

From boundary conditions (1), (2), (4), (5) and the first equation (7) and the second (8), we will get:

$$
\begin{gathered}
\left(\sigma_{\mathrm{yy}}-\mathrm{i} \sigma_{\mathrm{xy}}\right)^{+}-\left(\sigma_{\mathrm{yy}}-\mathrm{i} \sigma_{\mathrm{xy}}\right)^{-}==(\Phi(x)-\Omega(x))^{+}- \\
(\Phi(x)-\Omega(x))^{-}=0, x \in \widetilde{L}, \\
f^{+}(x)-f^{-}(x)=\left(\tilde{\mathrm{\kappa}} \Phi_{*}(x)-\Omega_{*}(x)\right)^{+} \\
\quad-\left(\tilde{\mathrm{\kappa}} \Phi_{*}(x)-\Omega_{*}(x)\right)^{-}=0 .
\end{gathered}
$$

By solving the linear conjugation (10) and considering the expression (9), we will have:

$$
\Omega(z)=\Phi(z)+\Gamma^{\prime}, \Omega_{*}(z)=\tilde{\kappa} \Phi_{*}(z)-(\tilde{\kappa}+1) \tilde{\Gamma}-\widetilde{\Gamma}^{\prime},
$$

If we introduce the function:

$$
\begin{aligned}
& F(z)=-2 \beta h^{2} \Phi(z)-m \tilde{\kappa} \Phi_{*}(z)-\beta h^{2} \Gamma^{\prime}++\frac{1}{2}\left\{i c^{\prime}+\right. \\
& \left.m\left[(\tilde{\kappa}+1) \tilde{\Gamma}-\widetilde{\Gamma}^{\prime}\right]\right\},
\end{aligned}
$$

then, as can be seen from the first condition (3) and (5), considering the first expressions (7) and (8), and (11), it will satisfy the boundary conditions:

$$
F^{+}(x)+F^{-}(x)=\left\{\begin{array}{l}
\sigma_{1}, x \in L_{1}, \\
0, x \in L,
\end{array}\right.
$$

where:

$\sigma_{1}=-2 h^{2} \beta \sigma_{0}-\mathrm{M}_{0}$.

Solution of the problem of linear conjugation (13) in the class of functions of limited plastic zones in the crack tips has the form: 


$$
\begin{aligned}
& F(z)=\frac{\sigma_{1} X_{1}(z)}{2 \pi i} \int_{L_{1}} \frac{d t}{X_{1}^{+}(t)(t-z)}= \\
& =\frac{\sigma_{1}}{\pi i} \ln \frac{d \sqrt{l^{2}-z^{2}}}{z \sqrt{d^{2}-l^{2}}+l \sqrt{d^{2}-z^{2}}}
\end{aligned}
$$

where:

$X_{1}(z)=\sqrt{z^{2}-d^{2}}$.

Taking into account (9), for a function $F(z)(12)$ at large $|z|$ we will have the expansion:

$F(z)=\frac{1}{2}\left(i c^{\prime}-\mathrm{M}_{\mathrm{y}}^{\infty}\right)-\beta h^{2} q+O\left(\frac{1}{z^{2}}\right)$.

Having converted the right part of (15) into a series at large $|z|$ and taking into account (16), we will get:

$\mathrm{c}^{\prime}=0$,

$-\frac{\left(\mathrm{M}_{\mathrm{y}}^{\infty}+2 \beta h^{2} q\right)}{2 \sigma_{1}}=\arccos \frac{l}{d}$.

From dependence (18) we determine length of the plastic zone $b$.

From boundary conditions:

$\sigma_{x y}^{+}+\sigma_{x y}^{-}=0, P^{+}+P^{-}=0, x \in \tilde{L}$,

considering (7), (8), (11), (17), we obtain a problem of linear conjugation:

$$
\begin{aligned}
& (\Phi(x)-\Phi(x))^{+}+(\Phi(x)-\bar{\Phi}(x))^{-}=0, x \in \tilde{L}, \\
& \left(\Phi_{*}(x)-\bar{\Phi}_{*}(x)\right)^{+}+\left(\Phi_{*}(x)-\bar{\Phi}_{*}(x)\right)^{-}=0, x \in \tilde{L},
\end{aligned}
$$

solving which in the class of functions of limited plastic zones at the crack tips, we will get:

$\bar{\Phi}(z)=\Phi(z), \Phi_{*}(z)=\bar{\Phi}_{*}(z)$,

Considering (8), (11), (17), (19), the second condition (3) and the first condition (4) and (5), will have

$$
\begin{gathered}
\left(\delta \Phi(x)+2 \Phi_{*}(x)-\frac{\mathrm{A}}{2}\right)^{+}-\left(\delta \Phi(x)+2 \Phi_{*}(x)-\frac{\mathrm{A}}{2}\right)^{-} \\
=0, x \in L,\left(\delta \Phi(x)+2 \Phi_{*}(x)-\frac{\mathrm{A}}{2}\right)^{+}+ \\
+\left(\delta \Phi(x)+2 \Phi_{*}(x)-\frac{\mathrm{A}}{2}\right)^{-}=0, x \in L_{1}
\end{gathered}
$$

where:

$\mathrm{A}=\delta \sigma_{0}-\delta \Gamma^{\prime}+2 \widetilde{\mathrm{K}}^{-1}\left(\mathrm{M}_{0} m^{-1}+(\tilde{\mathrm{\kappa}}+1) \tilde{\Gamma}+\widetilde{\Gamma}^{\prime}\right), \delta=$

$\frac{2(1-v)}{\mathrm{E} \alpha h}$,

Having solved the problem of linear conjugation (20) in the class of functions limited plastic zones at the crack tips, we get:

$\delta \Phi(z)+2 \Phi_{*}(z)-\frac{\mathrm{A}}{2}=0$. have:

Taking into account the expansion (9), based on (22) we will

$\mathrm{A}=2(\delta \Gamma+2 \tilde{\Gamma})$,

or

$-\frac{h^{2}}{3} \tilde{\gamma} \sigma_{0}+2 \mathrm{M}_{0}=2 \mathrm{M}_{y}^{\infty}-\frac{h^{2}}{3} \tilde{\gamma} q$, where: $\tilde{\gamma}=4 \gamma_{1}, \gamma_{1}=3+v /(\alpha(1+v))$,

To find $\sigma_{0}$ and $M_{0}$ firstly let us use Treska plasticity condition in the form of surface layer on the bottom base of the plate (Kushnir et al., 2003, Kyrjan et al. 2007):

$\sigma_{0}+3 \mathrm{M}_{0} /\left(2 h^{2}\right)=\sigma_{y}$,

where $\sigma_{y}$ - yield stress of the material of the plate.

Having solved the system of equations (24) and (25) for $\sigma_{0}$ and $M_{0}$, we will get:

$\tilde{\sigma}_{0}=\sigma_{0} / \sigma_{\mathrm{y}}=4(1+0.25 \tilde{\gamma} \tilde{q}-\tilde{\sigma}) /(4+\tilde{\gamma})$,

$\tilde{\mathrm{M}}_{0}=3 \mathrm{M}_{0} /\left(2 h^{2} \sigma_{\mathrm{y}}\right)=(4 \tilde{\sigma}+\tilde{\gamma}(1-\tilde{q})) /(4+\tilde{\gamma})$,

where:

$\tilde{\sigma}=\sigma_{\infty} / \sigma_{\mathrm{y}}, \sigma_{\infty}=3 \mathrm{M}_{\mathrm{y}}^{\infty} /\left(2 h^{2}\right), \tilde{q}=q / \sigma_{\mathrm{y}}$.

If we use plastic hinge condition (Kushnir et al., 2003, Kyrjan et al. 2007):

$\left(\frac{\sigma_{0}}{\sigma_{\mathrm{y}}}\right)+\frac{\mathrm{M}_{0}}{h^{2} \sigma_{\mathrm{y}}}=1$,

and solve the system of equations (24) and (28) for the same variables, we will get:

$\tilde{\sigma}_{0}=\sqrt{(\tilde{\gamma} / 12)^{2}+1-2 \tilde{\sigma} / 3+\tilde{q} / 6}-\tilde{\gamma} / 12$

$\widetilde{\mathrm{M}}_{0}=\tilde{\sigma}+0.25\left(\tilde{\gamma} \tilde{\sigma}_{0}-\tilde{q}\right)$.

If we solve the system of equations (12) and (22) for $\Phi(z)$ and $\Phi_{*}(z)$, then we will get:

$\Phi(z)=C_{5}+C_{6} F(z), \Phi_{*}(z)=C_{7}+C_{8} F(z)$,

where:

$C_{5}=C_{6}(\mathrm{~A} m \tilde{\mathrm{\kappa}}-4 \mathrm{~B}), C_{6}=2\left(\delta m \tilde{\mathrm{\kappa}}-4 \beta h^{2}\right)^{-1}, C_{7}=$ $0.5 C_{6}\left(\mathrm{~B} \delta-\mathrm{A} \beta h^{2}\right), C_{8}=-0.5 \delta C_{6}, \mathrm{~B}=-\beta h^{2} \Gamma^{\prime}+$ $m\left((\tilde{\mathrm{K}}+1) \tilde{\Gamma}+\widetilde{\Gamma}^{\prime}\right) / 2$.

To determine the contact force $N$ between the crack banks we take into account formulas (6), (7), (11) and (30). After the appropriate transformations we will get:

$N=\frac{\mathbf{M}_{\mathrm{y}}^{\infty}-2 h^{2} q \gamma_{1} / 3}{h\left(\beta+\gamma_{1} / 3\right)}$.

As so as $N>0$, such formulation of the problem exists when the following inequality is satisfied:

$q \leq \sigma_{\infty} / \gamma_{1}$,

which takes place, as shown in publications of Opanasovych et al. $(2008,2015)$ in a case, when there are no plastic zones in the crack tips.

Crack opening displacement $\delta$ in the crack tips on the lower base of the plate we will find by the formula:

$\delta=\int_{d}^{l} \partial_{\mathrm{x}}\left([v]-h\left[\partial_{\mathrm{y}} w\right]\right) \partial x$.

If we take into account (7), (8), (11), (19), (30), then after transformations from (33) we will get:

$\delta^{*}=\frac{\delta E}{\sigma_{y} l}=R \ln \frac{l}{d^{\prime}}$ 
where:

$R=32 R_{1}(1+\alpha) /(\pi \alpha(\tilde{\gamma}+12 \beta)) R_{1}=\widetilde{M}_{0}+3 \beta \tilde{\sigma}_{0}$.

If we introduce the relative length of plastic zone as $\varepsilon=b / l$, then formulas (18) and (34) will take forms

$\varepsilon=\sec \left(\pi(\tilde{\sigma}+3 \beta \tilde{q}) /\left(2 R_{1}\right)\right)-1, \delta^{*}=R \ln (1+\varepsilon)$.

Let us assume that parameter $\gamma$ linearly depends on $q$, that means it changes according to the law

$\gamma=\tilde{\gamma}_{1}\left(1-\alpha \gamma_{1} \tilde{q} / \tilde{\sigma}\right)$,

where $\tilde{\gamma}_{1}$ is the width of crack banks contact area at $p=q=0$ (Slepyan, 1995; Opanasovych, 2015).

It should be noted that for the case when we have only biaxial bending, i.e. $p=q=0$, we will get the results obtained in the article (Opanasovych, 2015).

\section{NUMERICAL ANALYSIS OF THE PROBLEM}

Numerical analysis of the problem was conducted for $\mathrm{v}=0.3$ and $\tilde{\gamma}_{1}=0.13$, and is presented in Fig. 2 and Fig. 3 , where the solid lines represent the results obtained at Treska plasticity conditions in the form of surface layer, and dash lines - obtained with the use of plastic hinge conditions.

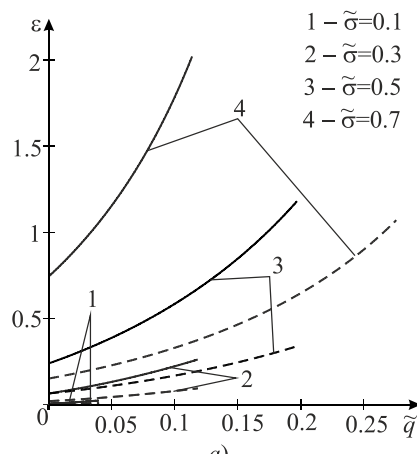

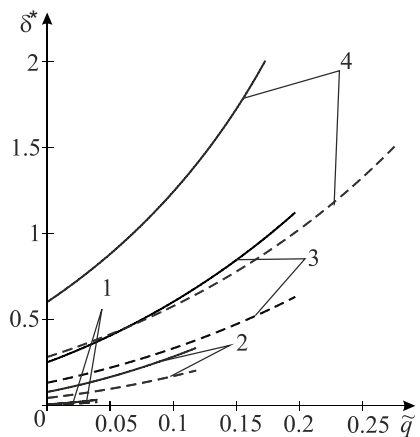

б)

Fig. 2. Graphical dependences of relative length of plastic zone $\varepsilon=b / l$ and dimensionless crack opening displacement $\delta^{*}=E \delta /\left(\sigma_{Y} l\right)$ on $\tilde{q}=q / \sigma_{Y}$
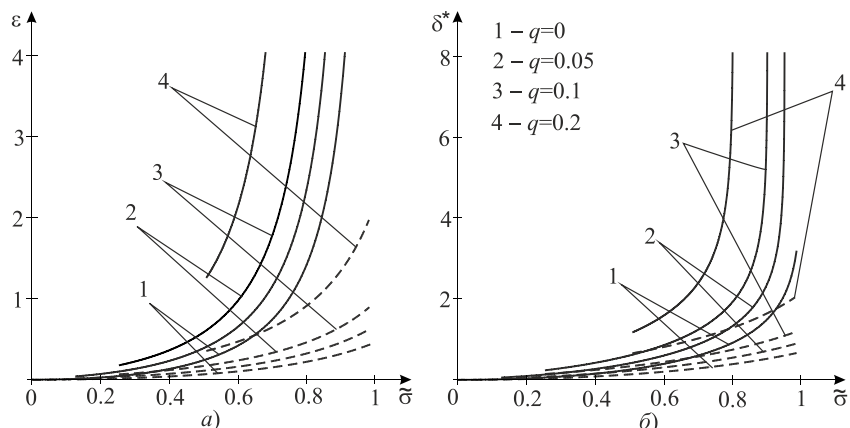

Fig. 3. Graphical dependences of relative length of plastic zone $\varepsilon=b / l$ and dimensionless crack opening displacement $\delta^{*}=E \delta /\left(\sigma_{Y} l\right)$ on $\tilde{\sigma}=\sigma_{\infty} / \sigma_{Y}$

On Fig. 2 the graphical dependences of relative length of plastic zone $\varepsilon=\mathrm{b} / \mathrm{l}$ (Fig. 2a) and dimensionless crack opening displacement $\delta^{*}=\mathrm{E} \delta /\left(\sigma_{\mathrm{Y}} \mathrm{l}\right.$ ) (Fig. $2 \mathrm{~b}$ ) on $\tilde{\mathrm{q}}=\mathrm{q} / \sigma_{\mathrm{Y}}$ are built.
Curve 1 is built at $\widetilde{\sigma}=\sigma_{\infty} / \sigma_{Y}=0.1(0 \leq \tilde{\mathrm{q}} \leq 0.0394)$, curve 2 - at $\widetilde{\sigma}=0.3(0 \leq \tilde{\mathrm{q}} \leq 0.1182)$, curve $3-$ at $\widetilde{\sigma}=0.5$ $(0 \leq \tilde{\mathrm{q}} \leq 0.197)$, curve $4-$ at $\widetilde{\sigma}=0.7(0 \leq \tilde{\mathrm{q}} \leq 0.2758)$.

On Fig. 3 the graphical dependences of relative length of plastic zone $\varepsilon=b / \mathrm{l}$ (Fig.3a) and dimensionless crack opening displacement $\delta^{*}=\mathrm{E} \delta /\left(\sigma_{\mathrm{Y}} \mathrm{l}\right)$ (Fig. $3 \mathrm{~b}$ ) on $\widetilde{\sigma}=\sigma_{\infty} / \sigma_{\mathrm{Y}}$ are represented. Curve 1 is built at $\tilde{\mathrm{q}}=\mathrm{q} / \sigma_{\mathrm{Y}}=0(0 \leq \widetilde{\sigma} \leq 1)$, curve 2 - at $\tilde{\mathrm{q}}=0.05(0.1269 \leq \widetilde{\sigma} \leq 1)$, curve 3 - at $\tilde{\mathrm{q}}=0.1$ $(0.2538 \leq \widetilde{\sigma} \leq 1)$, curve $4-$ at $\widetilde{q}=0.2(0.5077 \leq \widetilde{\sigma} \leq 1)$.

\section{CONCLUSIONS}

From the figures we can see, that with the increase of the external loads $\tilde{\mathrm{q}}=\mathrm{q} / \sigma_{\mathrm{Y}}$ and $\widetilde{\sigma}=\sigma_{\infty} / \sigma_{\mathrm{Y}}$ length of plastic zone in the crack tips and crack opening displacement increases. Also, this parameters, determined with the use of plasticity conditions in the form of surface layer, are higher than obtained by using plasticity condition in the form of plastic hinge. At $\widetilde{\sigma} \rightarrow 1$ a significant increase $\varepsilon$ and $\delta^{*}$ regardless on $\tilde{\mathrm{q}}$, is observed in case of using Treska plasticity condition in the form of surface layer, and it is not observed in the case of plastic hinge conditions. Our research shows, that the distributed bending moment at infinity when its vector is perpendicular to the crack, as well as the distributed force at infinity, does not affect length of the plastic zones and crack opening displacement.

\section{REFERENCES}

1. Alfavicka S.O. (2015), Bend of a plate with a through straight crack with plastic zones in its tip taking into account the crack faces contact and cold-hardening of material, Bulletin of Kyiv National Taras Shevchenko University. Series Sci. Vol. Special, 21-26

2. Boukellif R., Ricoeur A. (2014), Parameter identification for cracks in elastic plate structures based on remote strain fields, International Journal of Solids and Structures, 51 (11-12), 2123-2132.

3. Chen Y.Z., Lin X.Y. (2005), Periodic group crack problems in an infinite plate, International Journal of Solids and Structures, 42 (9-10), 2837-2850.

4. García-Collado A., Vasco-Olmo J.M., Díaz F.A. (2017), Numerical analysis of plasticity induced crack closure based on an irreversible cohesive zone model, Theoretical and Applied Fracture Mechanics, $89,52-62$.

5. Guimaraes S., Telles J.C.F. (2009), The method of fundamental solutions for fracture mechanics - Reissner's plate application, Engineering Analysis with Boundary Elements, 33 (10), 1152-1160.

6. Kushnir R.M., Nykolyshyn M.M., Osadchyk V.A. (2003), Elastic and elastic-plastic boundary state of membranes with defects, SPOLOM, Lviv.

7. Kyrjan V.I., Osadchuk V.A. Nykolyshyn M.M. (2007), Fracture mechanics of metal welded joints, SPOLOM, Lviv.

8. Musheviashvili N.I. (1966), Some basic problems of the mathematical elasticity theory, Science, Moscow.

9. Opanasovych V.K. (2007), Bend of plates with a through rectilinear crack taking into account the width of crack faces contact area, Scientific notes of the Lutsk Technical University: Interuniversity collection (on topic "Mechanical Engineering"), 20(2), 123-127.

10. Opanasovych V.K., Dorosh M. (2008), Combined bend with tension of plates weakened by two collinear cracks with contact of their faces, Bulletin of Lviv. Univ. Series meh.-mate, 68, 194-206.

11. Opanasovych V.K., Slobodjan M.S. (2015), Biaxial bending of isotropic plate with a through linear crack considering the width of the crack faces contact area and the presence of plastic zones in its tips, Mathematical methods and physico-mechanical fields, 58(4), 128-135 
12. Papargyri-Pegiou S., Theofanidis D., Webb T.W., Aifantis E.C. (1995), A simple approach for modelling the heterogeneity of crack tip plastic zones, Mechanics Research Communications, 22(3), 263-269.

13. Prawoto Y. (2012), How to compute plastic zones of heterogeneous materials: A simple approach using classical continuum and fracture mechanics, International Journal of Solids and Structures, 49 (15-16), 2195-2201.

14. Prusov I.A. (1975), The method of conjugation in the theory of plates, Publication Belarusian, University, Minsk.

15. Samaan M.F., Nassar M.E., Rashed Y.F. (2015), Taylor series fast multipole boundary element method for solution of Reissner's shear deformable plate bending problems, Engineering Analysis with Boundary Elements, 59, 23-35.

16. Shackyj I.P. (1989), On the contact of notch faces in plate at combined tension with bending, Physico-chemical Mechanics of materials, 25(2), 46-50.

17. Shackyj I.P. (1995), Boundary-equilibrium state of plates with collinear cracks at combined tension and bending, Reports of the NAS of Ukraine, 10, 62-64.
18. Shackyj I.P., Makovijchuk M.V. (2004), Interaction of cracks faces at the combined tension with bending of plate on elastic base, Mashinoznavstvo, 10, 62-68.

19. Shackyj I.P., Perepichka V.V. (2004), Boundary state of semiinfinite plate with edge crack at bend with tension, Physico-chemical Mechanics of materials, 40(2), 73-77.

20. Shi P.P. (2015), On the plastic zone size of solids containing doubly periodic rectangular-shaped arrays of cracks under longitudinal shear, Mechanics Research Communications, 67, 39-46.

21. Slepyan L.I. Dempsey J.P., Shekhtman I.I. (1995), Asymptotic solutions for crack closure in an elastic plate under combined extension and bending, Journal of the Mechanics and Physics of Solids. 43, 1727-1749.

22. Sulym H., Opanasowych W., Jacyk I. (2011), Bending of Reissner's plate containing cracks with the account of their faces contact zone width, Acta Mechanica et Automatica, 5(1), 85-93.

23. Unger D.J. (2007), Numerical plane stress elastic-perfectly plastic crack analysis under Tresca yield condition with comparison to Dugdale plastic strip model, Mechanics Research Communications, 34(4), 325-330.

24. Wang X.-F., Hasebe N. (2000), Bending of a thin plate containing a rigid inclusion and a crack, Engineering Analysis with Boundary Elements, 24(2), 145-153. 